\title{
Low Voltage SEM Characterization of Ceramic Aerogel
}

\author{
C.A. Wisner, ${ }^{*}$
}

* Materials Research Center and Department of Materials Science and Engineering, Missouri University of Science and Technology, Rolla, MO 65409, USA

Due to its many unique material properties, such as high mechanical strength, high thermal conductivity, exceptionally good resistance to oxidation, low coefficient of thermal expansion, and exceptional thermal stability is an extremely versatile material [1]. Efforts have been made to produce a ceramic aerogel incorporating $\mathrm{SiC}$. This material can be used as a catalytic support because it can withstand temperatures up to $1500^{\circ} \mathrm{C}[4]$.

Low voltage scanning electron microscopy (LVSEM) was used to characterize a ceramic aerogel with a Hitachi S4700 FEG-SEM. Images were captured using a $5 \mathrm{kV}$ accelerating voltage, from areas near the edge and the interior of the, Fig. 1. Initial images indicated that the edges of the sample differed from the center, as seen in Fig. 2 and Fig 3. There is a marked difference in the ase in the number of smaller particles and plate like particles in the interior image compared to the rim image. Energy dispersive $x$-ray spectrometry (EDS) was conducted to see if the differences were due to elemental concentrations or strictly from morphology. EDS analysis was conducted at $10 \mathrm{kV}$ at the middle and the rim and showed minor concentration differences in all the elements detected, $\mathrm{C}, \mathrm{N}, \mathrm{O}, \mathrm{Si}$, TABLE 1 . Because the sample surface was not smooth and the elements of concern are low energy, low voltage energy dispersive $\mathrm{x}$-ray spectrometry (LVEDS) was conducted at $5 \mathrm{kV}$ on the edge and interior of the ceramic aerogel cross section [2]. Low voltage energy dispersive x-ray spectrometry of the rim and interior again showed a difference in the elemental composition at the rim and interior. The difference in the carbon analysis between the 5 and $10 \mathrm{kV}$ analysis showed higher concentrations at $5 \mathrm{kV}$ at both the rim and interior.

The next step is to insure this difference in elemental concentration it is a property of the material and not an artifact of LVEDS. Artifacts arising from LVEDS include absorption of soft x-rays, and a contamination layer on surface [3]. Absorption was not considered a problem because the elements of interest in the sample were light elements. Small (2002) reported that contamination was not evident in his analysis performed in an FEG-SEM because the high vacuum in the sample chamber [2]. A carbon sample disk that had been exposed to atmosphere was analyzed at 5 and 10 $\mathrm{kV}$ to discern if there was a difference in carbon content from atmospheric contamination. The $5 \mathrm{kV}$ analysis reported 86.67 wt. \% C with the $10 \mathrm{kV}$ analysis reporting $90.08 \mathrm{wt}$. \% C. This information indicates that contamination was not an issue in the LVEDS and there is indeed an elemental difference across the cross section of the ceramic aerogel.

References

[1] X. Li et al., J Mater Sci. 44 (2009) 4661.

[2] J.A. Small, J. Res. Natl. Inst. Stand. Technol. 107 (2002) 564.

[3] E.A. Boyes, Mikrochim. Acta. 138 (2002) 230.

[4] The aid of Dr. N. Leventis and Anand Sadekar is gratefully acknowledged. 


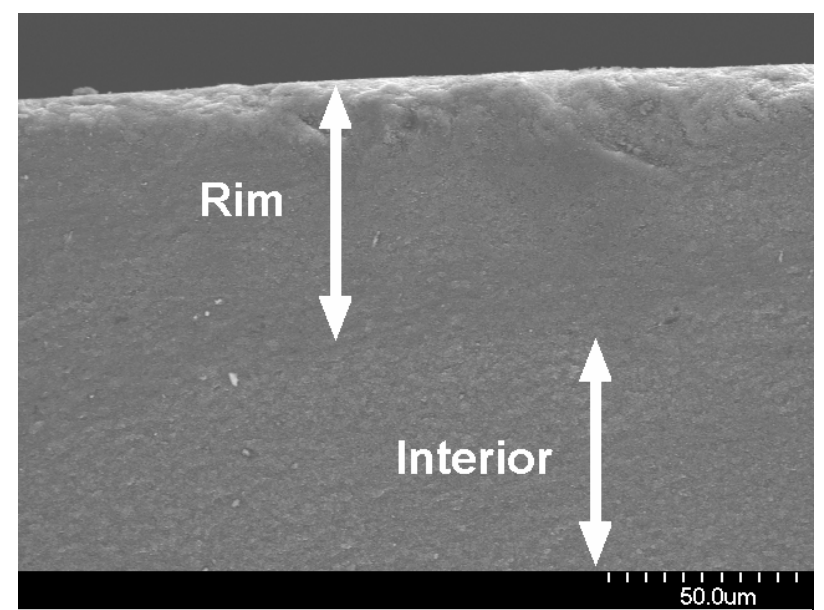

FIG. 1. Low mag of the rim and interior areas of the ceramic aerogel.

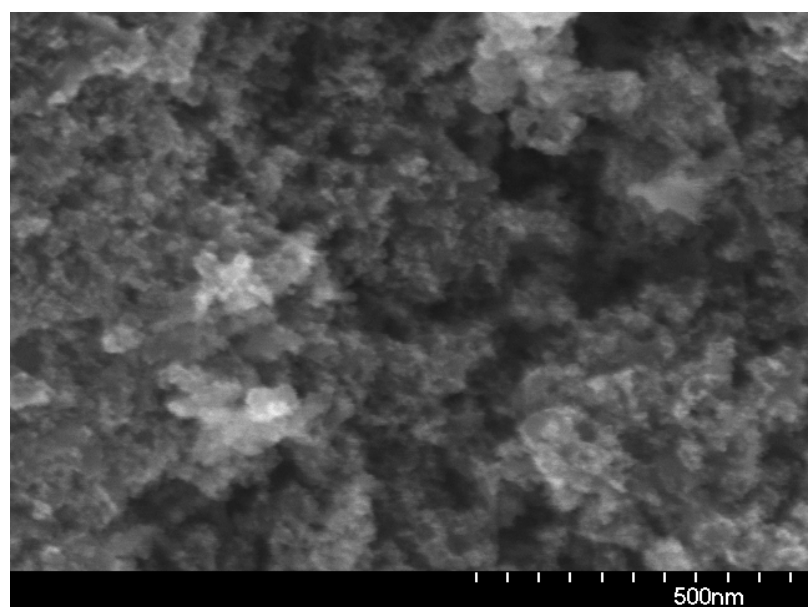

FIG. 2. High mag of the rim, small particles with a few "plates" present

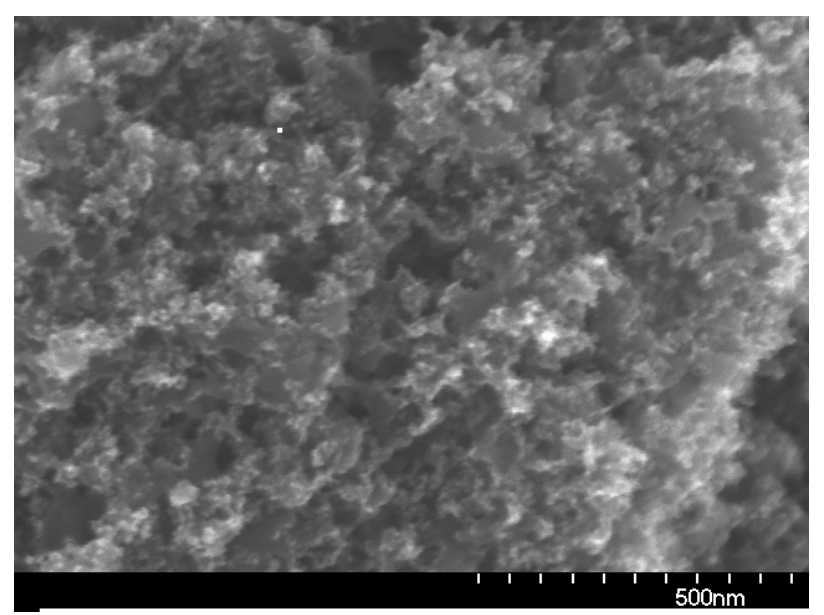

FIG. 3. High mag of the interior, small particles with more "plates" present

TABLE 1. Representative EDS results in wt. pct. at $5 \mathrm{kv}$ and $10 \mathrm{kV}$

\begin{tabular}{ccccc}
\hline & \multicolumn{2}{c}{$5 \mathrm{kV}$} & \multicolumn{2}{c}{$10 \mathrm{kV}$} \\
\hline Element & Rim & Interior & Rim & Interior \\
$\mathrm{C}$ & 49.40 & 53.42 & 42.30 & 50.27 \\
$\mathrm{O}$ & 25.54 & 23.17 & 24.93 & 28.68 \\
$\mathrm{Si}$ & 17.84 & 14.46 & 26.16 & 22.05 \\
$\mathrm{~N}$ & 7.21 & 8.95 & 4.91 & 6.72 \\
\hline
\end{tabular}

